\title{
Síndrome de Stevens-Johnson e Necrólise Epidérmica Tóxica em um hospital do Distrito Federal
}

\author{
Stevens-Johnson Syndrome and Toxic Epidermal Necrolysis in a hospital in Distrito Federal
}

Síndrome de Stevens-Johnson y necrólisis epidérmica tóxica en un hospital en Distrito Federal

\section{Mariane Ferreira Barbosa Emerick', Mayra Martins Toledo Rodrigues', Daniella Melo Arnaud Sampaio Pedrosa', Maria Rita Carvalho Garbi Novaes", Leila Bernarda Donato Gottems}

\author{
'Universidade Católica de Brasília, Centro de Ciências da Vida, Curso de Enfermagem. Brasília-DF, Brasil. \\ " Secretaria de Saúde do Distrito Federal, Fundação de Ensino e Pesquisa em Ciências da Saúde, \\ Faculdade de Medicina. Brasília-DF, Brasil.
}

Submissão: 29-01-2013 Aprovação: 22-09-2014

\section{RESUMO}

Objetivou-se analisar as características demográficas e clínicas dos clientes diagnosticados com Síndrome de Stevens Johnson (SSJ) e Necrólise Epidérmica Tóxica (NET), bem como identificar as ações dos profissionais de saúde para o manejo das Reações Adversas a Medicamentos (RAM) em um hospital público do Distrito Federal. Pesquisa descritiva, retrospectiva, com abordagem quantitativa. Dados coletados em todos os prontuários de 22 clientes internados de janeiro de 2005 a setembro de 2012. Análise mediante estatística descritiva. Houve aumento gradativo de casos, com maior número nos anos de 2007 e 2012. Dos casos analisados, 9 foram diagnosticados com NET e 7 com SSJ; predominaram as mulheres (14) e a faixa etária de 21 aos 40 anos (10); 21 obtiveram cura. Os fármacos associados a RAM mais frequentes foram os antiepilépticos (10). Observou-se fragilidade nos registros clínicos nos prontuários e nas ações de monitoramento de RAM no serviço estudado.

Descritores: Efeitos Colaterais e Reações Adversas Associados a Medicamentos; Erupção por Droga; Síndrome de Hipersensibilidade a Medicamentos.

\section{ABSTRACT}

This study aimed to analyze demographic and clinical aspects of patients diagnosed with Stevens Johnson Syndrome (SJS) and Toxic Epidermal Necrolysis (TEN), as well as identifying the actions of health professionals for the management of Adverse Drug Reactions (ADR) in a public hospital in Distrito Federal, Brazil. A descriptive and retrospective research was held, with quantitative approach. Data collected from all the records of 22 patients admitted with diagnosed with SJS and TEN, from January 2005 to September 2012. Data were analyzed using descriptive statistics. Of these cases, 9 were diagnosed with NET and 7, with SJS; there were more females (14); aged from 21 to 40 years (10); 21 were cured; the drugs more used were the antiepileptic ones (10). Fragility in clinical registers and in the actions to monitor the cases of ADR in this health service was observed.

Key words: Drug-Related Side Effects and Adverse Reactions; Drug Eruptions; Drug Hypersensitivity Syndrome.

\section{RESUMEN}

Este estudio tuvo como objetivo analizar aspectos demográficos y clínicos de clientes con diagnóstico de Síndrome de Stevens Johnson (SSJ) y Necrólisis Epidérmica Tóxica (NET), así como la identificación de las acciones de los profesionales de la salud para el manejo de reacciones adversas a medicamentos (RAM) en un hospital público del Distrito Federal. Se realizó investigación descriptiva, retrospectiva con enfoque cuantitativo. Datos recogidos de prontuarios clínicos de los 22 clientes ingresados con diagnóstico de SJS y NET, de enero de 2005 a septiembre de 2012. Fueron analizados utilizando estadística descriptiva. De estos casos, 9 fueron diagnosticados con NET, 7 con SJS; había más mujeres (14); edad entre 21 y 40 años (10); 21 se curaron; predominaran los antiepilépticos (10). Fue observado que hay fragilidad en registros clínicos en los prontuarios y en las acciones de monitoreo de las RAM en este servicio de salud.

Palabras clave: Efectos Colaterales y Reacciones Adversas Relacionados con Medicamentos; Erupciones por Medicamentos; Síndrome de Hipersensibilidad a Medicamentos. 


\section{INTRODUÇÃO}

A preocupação com o uso de medicamentos, seus riscos, toxicidade e efeitos colaterais é crescente no Brasil e no mundo, como reflexo da diversidade de formas de acesso e utilização de novos fármacos pela população. Os efeitos negativos da utilização de medicamentos expõem a população aos riscos de sofrerem Eventos Adversos (EA) que podem acarretar um aumento do número de hospitalizações e óbitos, bem como consequências epidemiológicas e econômicas acentuadas ${ }^{(1-2)}$.

O EA é definido como a ocorrência, em seres humanos, de qualquer efeito não desejado, decorrente da utilização de produtos sob vigilância sanitária, o qual pode ser evitável ou não ${ }^{(3-}$ 4). O evento adverso a medicamento (EAM) envolve diferentes situações, entre elas a reação adversa a medicamento (RAM) e a ineficácia terapêutica $(\mathrm{IT})^{(2)}$. A RAM é definida pela Organização Mundial da Saúde (OMS) como qualquer acontecimento danoso, não intencional e indesejado, ocorrido durante o uso de um medicamento empregado com doses terapêuticas habituais para tratamento, profilaxia ou diagnóstico ${ }^{(4)}$.

O monitoramento dos medicamentos, mesmo após a aprovação para comercialização pelas instituições de vigilância sanitária, tem sido objeto de diferentes diretrizes propostas por agências reguladoras que orientam os serviços de saúde a realizar a busca ativa de RAM. Estas atividades conformam as ações da farmacovigilância, que tem como finalidade detectar, avaliar, compreender e prevenir riscos de incidentes relacionados ao uso de medicamentos ${ }^{(3-6)}$.

A RAM pode se manifestar como reação leve, moderada ou de alta intensidade, por vezes requer hospitalização e provoca sequelas incapacitantes e letais. Os relatos de RAM são importantes para quantificar a incidência e para tratar rapidamente os clientes, de forma a minimizar a ocorrência de lesões graves e sequelas. Destacam-se, neste artigo, as RAM que se manifestam na forma de reações graves de hipersensibilidade cutânea denominadas de Síndrome de Stevens Johnson (SSJ) e a Necrólise Epidérmica Tóxica (NET) ${ }^{(7-9)}$.

A SSJ foi relatada em 1922, quando Stevens e Johnson descreveram dois clientes com erupções cutâneas generalizadas, febre contínua, mucosa oral inflamada e conjuntivite purulenta grave ${ }^{(8-9)}$. A SSJ é uma afecção inflamatória aguda, febril e autolimitada, com duração aproximada de duas a quatro semanas, que afeta a pele e a membrana mucosa ${ }^{(10-11)}$. A doença tem início súbito, com febre de $39-40^{\circ} \mathrm{C}$, dor, mal-estar, cefaleia, dor de garganta e na boca. Logo os sintomas evoluem com agravamento e o cliente pode apresentar pulso fraco e acelerado, respiração rápida, prostração e dores articulares ${ }^{(12-13)}$. Estomatite é um sintoma precoce, em geral com bolhas nos lábios, língua e mucosa oral, agravada pela pseudomembrana, perda de sangue, salivação e feridas que dificultam a alimentação e ingestão de bebidas ${ }^{(14-15)}$. O rosto, as mãos e os pés são invadidos por erupções hemorrágicas, vesículas, bolhas ou petéquias, com inflamação de alguns ou de todos os orifícios, tais como boca, nariz, conjuntiva, uretra, vagina e ânus. Essas lesões mostram-se espalhadas por todo o corpo. A vaginite pode ser erosiva grave. Não é incomum desenvolver pneumonia ${ }^{(15)}$. As bolhas geralmente não determinam descolamento epidérmico maior do que $10 \%$ da superfície corpórea(11,16).

A denominação Necrólise Epidérmica Tóxica (NET) foi introduzida por Lyell em 1956 ${ }^{(12)}$. O termo necrólise foi proposto para descrever a necrose isolada da camada epidérmica pelo destacamento da epiderme, onde praticamente não se vislumbram as alterações de caráter inflamatório, habitualmente presentes em situações de eritema tóxico ${ }^{(12,14)}$.

A SSJ e a NET ocorrem em aproximadamente duas a três pessoas por milhão/ano na Europa e Estados Unidos da América (EUA). Acometem clientes de todas as idades, raças e $\operatorname{sexos}^{(10)}$, sendo $20 \%$ crianças e adolescentes ${ }^{(13)}$. O pico de incidência, porém, está na segunda década de vida ${ }^{(15)}$. Indicadores do Brasil são escassos quanto à prevalência de SSJ e NET. Estima-se que a SSJ varie de 1,2 a 6 por milhões/ano e a NET varie de 0,4 a 1,2 por milhões/ano. Alguns fatores podem ser predisponentes, como comorbidades múltiplas e o uso de medicamentos para tratá-las, idade avançada, susceptibilidade genética, doenças com ativação imune e imunossupressão ${ }^{(14)}$.

A NET tem, como características iniciais, sintomas inespecíficos, influenza-símile, tais como febre, dor de garganta, tosse e queimação ocular, considerados manifestações prodrômicas que precedem em um a três dias o acometimento cutaneomucoso. Erupção eritematosa surge simetricamente na face e na parte superior do tronco, com extensão craniocaudal, provocando sintomas de descolamento epidérmico ou dor da pele. Em cerca de dois a cinco dias ou, por vezes, em questão de horas, ou, mais raramente, em cerca de uma semana, ocorre o estabelecimento completo da extensão do quadro cutâneo ${ }^{(9-12)}$.

O ápice do processo de desenvolvimento da NET ocorre com a desnudação da epiderme necrótica, a qual é destacada em verdadeiras lamelas ou retalhos, dentro das áreas acometidas pelo eritema de base ${ }^{(12)}$, podendo atingir uma superfície corporal menor que $30 \%{ }^{(13,15)}$.

A distinção entre a SSJ e a NET, do ponto de vista clínico, se realiza pela percentagem de área com erupções cutâneas ou passível de erosão com sinal de Nikolsky (SN) positivo, calculada em termos de superfície corporal ${ }^{(12)}$. Segundo a literatura, a SSJ acomete $\leq 10 \%$ da superfície corporal; a ocorrência simultânea de SSJ e NET pode comprometer 10 a $30 \%$ da epiderme; e a NET atinge área $\geq 30 \%$ da superfície corporal $^{(13-16)}$. O SN representa o deslocamento da camada superficial da epiderme mediante uma fricção ou trauma leve. Nos casos de SSJ e NET, o SN torna-se positivo sobre grandes áreas da pele. Muitos órgãos internos são acometidos pelo mesmo processo patológico que envolve a pele, determinando um espectro de manifestações sistêmicas ${ }^{(13,15)}$.

Os prognósticos dos casos de NET são avaliados segundo um escore de gravidade composto por sete parâmetros denominados SCORTEN: Idade > 40 anos, neoplasia, frequência cardíaca $>120 \mathrm{bpm}$, Descolamento da epiderme $>10 \%$, Ureia $>28 \mathrm{mg} / \mathrm{dL}$, Glicose $>252 \mathrm{mg} / \mathrm{dL}$, e Bicarbonato sérico $>20 \mathrm{mg} / \mathrm{dL}^{(12-14)}$.

Os fármacos mais comumente associados ao desencadeamento da reação são as sulfonamidas, anti-inflamatórios, penicilina, barbitúricos, alopurinol, antiepilépticos e vacinas ${ }^{(8,11)}$. Acredita-se que o mecanismo de desenvolvimento da SSJ e da 
NET esteja associado a reações de hipersensibilidade mediadas por células T citotóxicas, além de outros fatores predisponentes a RAM, como alterações no metabolismo de fármacos, deficiência nos mecanismos de detoxificação de metabólitos intermediários reativos e a predominância de um genótipo de acetiladores lentos ${ }^{(12)}$.

As complicações mais frequentes da SSJ e da NET, segundo a literatura, são a sepse, que, quando não reconhecida e tratada, pode levar ao óbito; e a ceratoconjuntivite, que pode prejudicar a visão e resultar em retração conjuntival, cicatrização e lesão da córnea ${ }^{(16)}$. Entre as sequelas mais comuns na fase tardia da NET, citam-se a hiper ou hipopigmentação da pele $(62,5 \%)$, as distrofias das unhas $(37,5 \%)$ e as complicações oculares, dentre as quais se destacam: olhos secos $(46,0 \%)$, triquíase $(16,0 \%)$, simbléfaro $(14,0 \%)$, distiquíase $(14,0 \%)$, perda da visão $(5,0 \%)$, entrópio $(5,0 \%)$, ancilobléfaro $(2,0 \%)$, lagophthalmos $(2,0 \%)$ e ulceração da córnea $(2,0 \%)$. Cicatrizes hipertróficas têm prevalência baixa. Complicações, com envolvimento de mucosas, ocorrem em 73,0\% dos clientes na fase aguda da doença; e as sequelas atingem, principalmente, a mucosa oral e esofagiana e se estendem menos para a mucosa pulmonar e genital ${ }^{(15-16)}$. Todas as possíveis complicações e sequelas precisam ser cuidadosamente observadas durante o tratamento, pela equipe de saúde.

As reações adversas caracterizadas como SSJ e NET ainda são consideradas pouco frequentes na literatura e no cotidiano dos serviços de saúde. Para o manejo clínico adequado, pelos profissionais de saúde, esses agravos devem ser compreendidos quanto ao mecanismo de ação e fatores desencadeadores, para que possam ser objeto de ações preventivas, diagnóstico precoce, tratamento oportuno e obtenção do melhor prognóstico.

Neste sentido, os objetivos deste estudo foram: analisar as características demográficas e clínicas dos clientes diagnosticados com SSJ e NET internados entre 2005 e 2012, bem como identificar as ações dos profissionais de saúde para o manejo das RAM em um hospital público do Distrito Federal.

\section{MÉTODO}

O estudo foi descritivo transversal e retrospectivo, com abordagem quantitativa. Estudos descritivos quantitativos são apropriados para investigar fenômenos pouco conhecidos em suas particularidades, pois possibilitam que o pesquisador observe, descreva e documente os vários aspectos do objeto, sem manipulação de variáveis ou procura pela relação de causalidade. Por outro lado, os estudos transversais são aplicáveis às investigações das relações causa e efeito quando não há a necessidade de saber o tempo de exposição de uma causa para gerar o efeito. Em outras palavras, a informação acerca da exposição e do agravo são coletadas ao mesmo tempo ${ }^{(17-18)}$. Complementarmente, a classificação de estudo retrospectivo foi empregada para caracterizar as variáveis associadas aos fatores causais da SSJ e NET identificadas após a ocorrência das doenças.

Os dados foram coletados por meio de análise documental dos relatórios de enfermagem e dos registros da equipe de saúde nos prontuários de todos os $(\mathrm{n}=22)$ clientes com diagnóstico de SSJ e NET hospitalizados na Unidade de Terapia Intensiva (UTI) e/ou Unidade de Tratamento de Queimados (UTQ), em associação com as unidades de dermatologia, plástica ou pediatria, de um hospital público do Distrito Federal.

Os relatórios de enfermagem usados como fonte complementar de informações neste estudo foram livros com folhas pautadas e numeradas, utilizados para o registro de intercorrências clinicas e administrativas que precisam receber maior atenção pela equipe e em especial, pelas chefias das unidades. Os relatórios de enfermagem contribuíram para a identificação de casos de SSJ e NET, para facilitar a busca pelos prontuários físicos nos arquivos, nos períodos anteriores à implantação do prontuário eletrônico do paciente no hospital, bem como para identificar as ações de vigilância das RAM. Por meio desses relatórios foi possível incluir no estudo todos os clientes que tiveram registro de hospitalização no período de janeiro de 2005 a dezembro de 2012 e que permaneceram internados por período superior a 24 horas.

Os dados como idade e sexo, diagnóstico da enfermidade de base, superfície corporal comprometida, fármacos usados pelos clientes quando do surgimento dos primeiros sinais e sintomas, complicações, tratamento e desfechos foram coletados no período de janeiro a julho de 2012. Esses foram organizados no software Excel (2007) e analisados mediante estatística descritiva.

Para a classificação da superfície corporal com descolamento epidérmico, utilizou-se a Regra dos Nove de Wallace. Essa regra emprega valor igual a nove ou múltiplo de nove às partes atingidas do corpo do adulto, da seguinte forma: cada braço, coxa e perna equivalem a 9,0\% da superfície corporal, assim como a cabeça, o tórax e o abdome; o dorso equivale a 18,0\%. A soma de todas estas partes totalizam $99,0 \%$. O 1,0\% restante é o períneo. Para pequenas regiões, usa-se uma comparação da área atingida com a palma da mão que equivale a 1,0\% da superfície corporal ${ }^{(13)}$. Em crianças de até 1 ano cabeça e pescoço são 19,0\% e cada membro inferior 13,0\%. Em crianças de 1-10 anos cabeça e pescoço são 19,0\% menos a idade e, cada membro inferior 19,0\% mais (idade dividido por 2).

As informações clinicas registradas nos prontuários foram comparadas aos critérios usados para o diagnóstico médico de SSJ e NET em relação à superfície corporal comprometida. Os critérios indicam que: SSJ acomete $\leq 10,0 \%$ da superfície corporal; SSJ e NET, simultaneamente, acometem 10,0-30,0\%; e a NET compromete área $\geq 30,0 \%{ }^{(12-14)}$. Embora a literatura recomende outros fatores de risco e critérios para avaliação da gravidade, sumarizados no SCORTEN, neste estudo foram coletados somente a idade e o descolamento epidérmico, devido à fragilidade nos registros clínicos nos prontuários e por se tratar de estudo retrospectivo.

Os fármacos associados ao desencadeamento da RAM foram classificados de acordo com os níveis anatômico (Nível 1) e terapêutico (Nível 2) da Anatomic Therapeutic Clinical (ATC), recomendada pela Organização Mundial de Saúde ${ }^{(19)}$.

O projeto de pesquisa foi aprovado pelo Comitê de Ética em Pesquisa da Fundação de Ensino e Pesquisa em Ciências da Saúde, Secretaria Estadual de Saúde, Brasília, Distrito Federal (CEP/FEPECS), sob o número de protocolo (164/2012). Foram preservados o sigilo e a confidencialidade dos dados coletados dos prontuários. 


\section{RESULTADOS}

No ano de 2005, a UTQ do hospital estudado recebeu 1 cliente com SSJ. Nos anos de 2007 e 2012, houve 5 e 7 diagnósticos confirmados, respectivamente (Tabela 1). Somente em 7 casos foi possível identificar o número de dias decorridos desde o início do uso do medicamento até o aparecimento dos principais sintomas, que variou de 2 a 20 dias, com média de 8 dias.

Tabela 1 - Incidência de SSJ e NET em hospital público de Brasília, no período de 2005 a 2012, Brasília-DF, 2012

\begin{tabular}{lccccccccc}
\hline Ano & 2005 & 2006 & 2007 & 2008 & 2009 & 2010 & 2011 & 2012 & Total \\
\hline No de casos $^{1}$ & 1 & 2 & 5 & 1 & 2 & 3 & 1 & 7 & 22 \\
\hline
\end{tabular}

Dos 22 prontuários analisados (eletrônicos e físicos), 7 casos foram diagnosticados como SSJ, 9 como NET e em 6 casos não houve diferenciação entre SSJ ou NET (Tabela 2). As unidades em que os clientes estiveram hospitalizados foram: Unidade de Queimados (13), Unidade Terapia Intensiva e de queimados (4), Unidade de Terapia Intensiva (2), Dermatologia (1), Unidade de Cirurgia Plástica (1), Pediatria (1). O tempo de internação em número de dias, identificado em 18 casos, variou de 2 a 47 dias, com uma média de 15,17 dias ( \pm desvio padrão 12,29\%). Em relação ao sexo, predominou o feminino (14). A faixa etária mais acometida foi a de 11 a 40 anos, com 14 dos casos, variando de 11 a 86 anos, com uma média de idade de 37,6 anos.

A área total da superfície corporal com descolamento epidérmico constava em 20 dos 22 prontuários analisados, sendo mais frequente (15) o comprometimento de áreas $\geq 31 \%$ (Tabela 2). As lesões provocadas pelo descolamento epidérmico foram classificadas em queimaduras de primeiro, segundo e terceiro grau, por se tratar de um hospital especializado nesse tipo de tratamento. Dos 16 prontuários com esta informação, 9 foram classificados como lesões de segundo grau, 6 com lesões superficiais e 1 caso com lesão profunda. A gravidade e intensidade da RAM, segundo os registros nos prontuários, foi leve em 5 dos casos, moderada em 16 e grave em 1 . O cliente classificado como RAM grave, com idade de 70 anos, evoluiu para o óbito. Esse teve cerca de $90 \%$ da área total de superfície corporal com descolamento epidérmico, segundo os registros.

O tratamento tópico mais utilizado nos clientes com descolamento epidérmico incluídos nesta pesquisa foram os Ácidos Graxos Essenciais (AGE).

Em 21 dos casos estudados, foi possível identificar o fármaco associado ao desencadeamento da RAM e classificá-lo de acordo com os níveis anatômico (Nível 1) e terapêutico (Nível 2), da Anatomic Therapeutic Clinical (ATC). Seguindo esta categorização, identificaram-se os medicamentos com ação sobre o Sistema Nervoso (15), anti-infecciosos gerais para uso sistêmico (4) e outros (3). Em relação à ação anátomo-terapêutica, os antiepilépticos (10), os antibióticos (3) e os analgési$\cos (1)$, usados isoladamente, foram os possíveis desencadeadores da RAM. Nos demais 7 casos, foi observado que houve associação de fármacos de diferentes classificações ATC.

Em apenas dois casos analisados identificou-se que a RAM foi decorrente de automedicação, com associação entre fármacos com efeito analgésico e para terapia sintomática da gripe: Dipirona simples e associada com desclorfeniramina, e broncodilatador (fenoterol) associado à corticosteroide (Prednisona) (Quadro 1). Nos demais casos, os medicamentos foram prescritos pelo médico.

Tabela 2 - Caracterização demográfica e clínica dos clientes diagnosticados SSJ e NET em hospital público do DF no período de 2005 a 2012, Brasília-DF, 2012

\begin{tabular}{|c|c|c|}
\hline Variáveis & & $f$ \\
\hline \multirow[t]{3}{*}{ Reação adversa $(n=22)$} & SS & 7 \\
\hline & NET & 9 \\
\hline & Sem diferenciação & 6 \\
\hline \multirow[t]{2}{*}{ Sexo $(n=22)$} & Feminino & 14 \\
\hline & Masculino & 8 \\
\hline \multirow[t]{6}{*}{ Faixa etária em anos $(n=22)$} & $\leq 11-20$ & 4 \\
\hline & $\leq 21-30$ & 5 \\
\hline & $\leq 31-40$ & 5 \\
\hline & $\leq 41-50$ & 1 \\
\hline & $\leq 51-59$ & 4 \\
\hline & $\geq 60$ & 3 \\
\hline Percentual de área total com & 0 a 10,0 & 1 \\
\hline descolamento epidérmico & 11,0 a 30,0 & 4 \\
\hline \multirow[t]{2}{*}{$(\mathrm{n}=20)$} & $\geq 31,0$ & 15 \\
\hline & $\mathrm{SI}$ & 2 \\
\hline \multirow[t]{4}{*}{ Dias de internação $(n=18)$} & $2-5$ & 3 \\
\hline & $6-10$ & 5 \\
\hline & $10-19$ & 5 \\
\hline & $\geq 20$ & 5 \\
\hline \multirow[t]{6}{*}{ Setores de internação $(n=22)$} & UTQ & 13 \\
\hline & UTQ/UTI & 4 \\
\hline & UTQ/Dermatologia & 1 \\
\hline & UTQ/Plástica & 1 \\
\hline & UTQ/Pediatria & 1 \\
\hline & UTI & 2 \\
\hline
\end{tabular}

Destaca-se ainda a fragilidade nos registros das informações demográficas e clínicas nos prontuários. Isso dificultou a obtenção de dados mais precisos sobre o tempo decorrido entre o uso do medicamento e o aparecimento dos sinais e sintomas, tempo de internação, gravidade dos casos por meio do SCORTEN, os possíveis fármacos que desencadearam a EA, bem como o acompanhamento do cliente após a alta.

Em relação às ações dos profissionais de saúde para o manejo das RAM, não foram encontrados, no período, registros sistematizados de notificação, tampouco existia no hospital, um setor responsável pela Gerência de Risco e/ou de Farmacovigilância. 
Quadro 1 - Fármacos relacionados ao desencadeamento da SSJ e NET em casos tratados em um hospital público do Distrito Federal, no período de 2005 a 2012, Brasília-DF, 2012

\begin{tabular}{|c|c|c|c|c|}
\hline Nível anatômico & Fármacos & $\mathbf{N}^{\circ}$ & Grupos & Cód. ATC \\
\hline \multirow[t]{5}{*}{ Sistema Nervoso (N) } & Fenobarbital & 4 & Antiepiléptico & N03 \\
\hline & Fenitoína & 3 & Antiepiléptico & N03 \\
\hline & Carbamazepina & 2 & Antiepiléptico & N03 \\
\hline & Lamotrigina & 1 & Antiepiléptico & N03 \\
\hline & Dipirona & 1 & Analgésico & N02 \\
\hline \multirow{3}{*}{$\begin{array}{l}\text { Anti-infecciosos gerais } \\
\text { de uso sistêmico }(J)\end{array}$} & Penicilina benzatina & 1 & Antibiótico & J01 \\
\hline & Amoxicilina & 1 & Antibiótico & J01 \\
\hline & $\begin{array}{l}\text { Trimetoprima e sulfametoxazol, associados a } \\
\text { Penincilina benzatina e Eritromicina }\end{array}$ & 1 & Antibiótico & J01 \\
\hline $\begin{array}{l}\text { Sistema } \\
\text { musculoesquelético }(\mathrm{M})\end{array}$ & Alopurinol & 1 & Antigotoso & M04 \\
\hline \multirow{7}{*}{$\begin{array}{l}\text { Associação de } \\
\text { fármacos de diferentes } \\
\text { classificações ATC }\end{array}$} & $\begin{array}{l}\text { Dipirona associado ao Resfenol* (paracetamol/ } \\
\text { maleato de clorfenamina/cloridrato de fenilefrina)** }\end{array}$ & 1 & Analgésico e antigripal & N02 \\
\hline & Rifampisina e Dapsona associado à Prednisona*** & 1 & $\begin{array}{l}\text { Antibiótico e } \\
\text { corticosteroide }\end{array}$ & J04 e H02 \\
\hline & Fenitoína associado à Carbamazepina & 1 & $\begin{array}{l}\text { Anticonvulsivante e } \\
\text { Antiepiléptico }\end{array}$ & N03 \\
\hline & Fenobarbital associado à Metildopa & 1 & $\begin{array}{l}\text { Antiepiléptico e } \\
\text { Anti-hipertensivo }\end{array}$ & N03 e C02 \\
\hline & Enalapril associado à Dipirona & 1 & $\begin{array}{l}\text { Anti-hipertensivo e } \\
\text { analgésico }\end{array}$ & C09 e N02 \\
\hline & Fenoterol associado à Prednisona** & 1 & $\begin{array}{l}\text { Broncodilatador e } \\
\text { corticosteroide }\end{array}$ & H02 e R03 \\
\hline & Prontuário sem informação & 1 & - & - \\
\hline Total & \multicolumn{4}{|c|}{22} \\
\hline
\end{tabular}

*Produto para terapia sintomática da gripe, classificado através da Relação de Medicamentos de Referência - Agência Nacional de Vigilância Sanitária, 2010; **Associação através de automedicação, dados do prontuário;

***Poliquimioterapia para tratar Hanseníase.

\section{DISCUSSÃO}

Um dos fatores de risco avaliado neste trabalho foi a idade. A prevalência de clientes diagnosticados com SSJ e NET foi maior na faixa etária de 11 a 40 anos $(n=14)$. Os dados encontrados contrapõem-se à literatura, que afirma que o pico de incidência está na segunda década de vida ${ }^{(13-15)}$. Por outro lado, 3 dos 22 casos analisados eram idosos, com idade igual ou acima de 60 anos (Tabela 2), faixa etária mais acometida por doenças crônico-degenerativas e, portanto, com maior predisposição e incidência de RAM devido a polifarmacoterapia ${ }^{(14,20-22)}$. Predominou o sexo feminino, consonante com outros estudos cujos achados indicam a maior incidência entre as mulheres.

Quanto à gravidade da RAM, observou-se que somente o caso de uma cliente idosa, com 70 anos de idade e cerca de
90,0\% de área com descolamento epidérmico e diagnóstico de NET, evoluiu para o óbito. Este dado está coerente com a literatura quanto à maior probabilidade de mortalidade em ido$\operatorname{sos}^{(14,20-22)}$. A literatura aponta que a mortalidade nos casos de SSJ ocorre em aproximadamente $10,0 \%$, e nos casos de NET, entre $30,0 \%$ e $70,0 \%$ dos $\operatorname{casos}^{(16)}$. A mortalidade causada pela SSJ e NET aumenta com a idade e de acordo com a região do corpo afetada, por serem agravos que provocam reação cutânea grave que demanda diagnóstico rápido e adequado ${ }^{(16)}$.

Entre os clientes estudados, 20 foram tratados na UTQ e 2 na UTI, sinalizando que o manejo clínico foi adequado neste serviço, conforme sugere a literatura especializada ${ }^{(13,20)}$.

Os fármacos predominantes no desencadeamento da RAM neste estudo foram aqueles que atuam no Sistema Nervoso (N), especialmente os anticonvulsivantes (fenobarbital, fenitoína e 
carbamazepina), e os anti-infecciosos gerais para uso sistêmico (J), com destaque para os antibióticos. Estes achados corroboram os dados obtidos na literatura que informam que os fármacos mais frequentemente associados a SSJ e a NET foram os antibióticos, com destaque para as penicilinas, as sulfas e os anticonvulsivantes, em especial a fenitoína e a carbamazepina $^{(8,11,22)}$. A identificação do fármaco que desencadeou a RAM é essencial para a definição da ação terapêutica inicial, tendo em vista que a suspensão do uso deve ser imediata, seguida do tratamento tópico das lesões causadas ${ }^{(20-22)}$.

A literatura disponível ainda não é suficiente para explicar o nexo causal entre o uso de medicamentos e o desenvolvimento de RAM, mas aponta que a imunossupressão, a idade avançada e as crianças estão mais vulneráveis. Todavia, há registros de indivíduos previamente saudáveis que fizeram uso de medicamentos como antibióticos e anti-inflamatórios não corticoides e desenvolveram reações adversas ${ }^{(10,13-15,22)}$. Neste sentido, os resultados deste estudo reforçam a necessidade de mais investigações sobre as RAM, com ênfase nos fatores sociodemográficos, clínicos e farmacológicos associados ao desenvolvimento da SSJ e NET.

O serviço estudado se caracteriza por ser um hospital de referência no tratamento de queimados e por isto utiliza a Regra dos Nove de Wallace para determinação da Área Total de Superfície Queimada (ATSQ) ${ }^{(23)}$ também para avaliar a área atingida com descolamento de epiderme nos casos de SSJ e NET. Esta forma de classificação, embora pouco usada nesses casos, estava registrada em 20 prontuários e se constitui em parâmetro para o diagnóstico diferencial entre SSJ e NET. Entretanto, observou-se nos registros dos prontuários que o cálculo de superfície atingida com descolamento epidérmico não foi adequadamente utilizado para diferenciar os casos de SSJ e NET ${ }^{(13-16)}$. Cita-se que 7 casos foram diagnosticados com SSJ, mas somente 1 cliente teve menos de $10 \%$ da superfície corporal atingida. Outros 4 casos tiveram uma superfície corporal com descolamento epidérmico entre 10 e $30 \%$ e deveriam ter sido diagnosticados com SSJ e NET simultâneos $^{(13-15)}$. Porém, nenhum caso foi diagnosticado dessa forma. Além disso, 15 clientes tiveram mais de $30 \%$ da superfície corporal com descolamento epidérmico, mas apenas 9 foram diagnosticados com NET.

Estes dados confirmam a necessidade de maior produção e disseminação de informações sobre a incidência de RAM, em especial as que se caracterizam por erupções cutâneas leves, moderadas e graves, para aumentar a capacidade de prevenção, diagnóstico e tratamento ${ }^{(4-9)}$. Assim, ratifica-se a necessidade de avaliação clínica especializada e a vigilância rigorosa de EA, EAM e RAM para prevenir o sofrimento humano induzido por medicamentos e diminuir custos financeiros associados com efeitos adversos inesperados ${ }^{(4-6)}$.

Não foram encontrados outros registros para o monitoramento de RAM, conforme proposto pela farmacovigilância, especialmente as notificações dos EA a setores próprios dentro do hospital, no período estudado. Estas informações associadas à fragilidade nos dados registrados nos prontuários reforçam a necessidade de maior disseminação de informações e de capacitação dos profissionais de saúde, seja para a notificação dos casos, seja para maior critério no diagnóstico e seguimentos dos casos após a alta. Além disso, é necessário investir na melhoria da documentação dos casos, de forma a incluir nela dados completos, confiáveis e de fácil acesso, especialmente aos profissionais responsáveis pelas ações de farmacovigilância ${ }^{(5-6)}$.

\section{CONCLUSÃO}

Os resultados mostraram que no período de 2005 a 2012 foram tratados neste hospital 9 casos de NET, 7 de SSJ e outros 6 sem o diagnóstico diferencial. Predominou o sexo feminino e a faixa etária de 21 aos 40 anos. A maioria dos fármacos foi prescrita, sendo os antiepilépticos a principal classe farmacológica associada a RAM, seguida dos antibióticos. Contudo, observou-se neste estudo divergência nos diagnósticos de SSJ e NET considerando-se as recomendações da literatura. Apesar de não trazer prejuízo ao tratamento, o diagnóstico de grande parte dos clientes, não considerou a superfície corporal com descolamento epidérmico, parâmetro importante de diferenciação entre SSJ e NET. As lesões por descolamento epidérmico causam grande prejuízo à qualidade de vida do cliente com SSJ e NET, por provocarem dor intensa e deixarem-no vulnerável a processos infecciosos.

Os casos tratados nesse período tiveram, em sua maioria (21), desfechos favoráveis, com alta para o cliente, e apenas 1 óbito. Entre os prontuários que continham a informação, 9 sofreram lesões epidérmicos de $2^{\circ}$ grau. Todos os casos foram tratados na UTQ e/ou na UTI.

Neste estudo foram encontradas limitações nos registros dos prontuários físicos e eletrônicos dos clientes, principalmente relacionadas aos dados demográficos, clínicos, sequelas e ao acompanhamento ambulatorial posterior à alta. Não foram encontradas informações sobre a notificação de RAM. Essas informações são importantes, dadas as características desses agravos, que apesar de raros, geram um forte impacto econômico e social, com risco potencial de provocar cegueira, modificações na pele e sobrecarga psicológica em população de perfil sóciodemográfico diverso.

\section{REFERÊNCIAS}

1. Oliveira JR, Xavier RMF, Santos Junior AF. Eventos adversos notificados ao Sistema Nacional de Notificações para a Vigilância Sanitária (NOTIVISA): Brasil, estudo descritivo no período 2006 a 2011. Epidemiol Serv Saúde. 2013;22(4):671-8
2. Lima PF, Cavassini ACM, Silva FAT, Kron MR, Gonçalves SF, Spadotto A, et al. Queixas técnicas e eventos adversos a medicamentos notificados em um hospital sentinela do interior de São Paulo, 2009-2010. Epidemiol Serv Saúde. 2013;22(4):679-86. 
3. Agência Nacional de Vigilância Sanitária (BR). Resolução-RDC no 23, de 4 de abril de 2012. Dispõe sobre a obrigatoriedade de execução e notificação de ações de campo por detentores de registro de produtos para a saúde no Brasil [Internet]. Diário Oficial da União 09 abr 2012 [acesso em 13 de julho de 2014];Seção 1. Disponível em: http://portal.anvisa.gov.br/wps/wcm/connect/578fd4004aeed $11 \mathrm{fba}$ 82bfa337abae9d/Resolucao_RDC_n_23_de_04_de_abril_ de_2012.pdf?MOD=AJPERES

4. World Health Organization. The conceptual framework for the International Classification for Patient Safety. Geneva: World Health Organization [Internet]. 2009 [cited 2014 July 13]. Available from: http://www.who.int/patientsafety/taxonomy/icps_chapter3.pdf

5. MotaDM. Investigaçãoem farmacoepidemiologiadecampo: uma proposta para as ações de farmacovigilância no Brasil. Rev Bras Epidemiol [Internet]. 2011 [acesso em 13 de julho de 2014];14(4):565-79. Disponível em: http://www.scielo.br /scielo.php?pid = S1415-790X2011000400004\&script =sci_arttext

6. Organização Mundial da Saúde. A importância da farmacovigilância: monitorização da segurança dos medicamentos [Internet]. Brasília (DF): Organização Pan-Americana da Saúde; 2005 [acesso em 13 julho de 2014]. Disponível em: http://bvsms.saude.gov.br/bvs/publicacoes/importancia.pdf

7. Gur S, Agnihotri R. Phenobarbital induced Stevens-Johnson syndrome in a child. Indian J Pharmacol. 2012;44(4):531-2.

8. Franca MD, Lima JPG, Freitas D, Cunha M, Gomes JAP. Estudo dos achados oculares na Síndrome de Stevens-Johnson em pacientes de centro de referência de atendimento terciário. Arq Bras Oftalmol. 2009;72(3):370-4.

9. Bulisani ACP, Sanches GD, Guimarães HP, Lopes RD, Vendrame LS, Lopes AC. Síndrome de Stevens-Johnson e Necrólise Epidérmica Tóxica em Medicina Intensiva. Rev Bras Ter Intensiva [Internet]. 2006 [acesso em 13 julho de 2014];18(3):292-7. Disponível em: http://www.scielo.br/ scielo.php?pid =s0103-507×2006000300012\&script =sci_arttext

10. Álvarez LMP, Salcedo MAS, Pacheco DLS. Síndrome de Stevens-Johnson. Presentación de 1 caso. Rev Cuba Pediatr. 2011;73(4):240-4.

11. lurk LK, Oliveira AF, Gragnani A, Ferreira LM. Evidências no tratamento de queimaduras. Rev Bras Queimaduras [Internet]. 2010 [acesso em 13 julho de 2014];9(3):95-9. Disponível em: file:///C:/Users/Cliente/Downloads/v9n3a04.pdf

12. Oliveira A, Sanches M, Selores M. O espectro clínico Síndrome de Stevens-Johnson e Necrólise Epidérmica Tóxica. Acta Med Port [Internet]. 2011 [acesso em 13 julho de
2014];24(S4):995-1002. Disponível em: file:///C:/Users/ Cliente/Downloads/1567-2216-1-PB.pdf

13. Cabral L, Diogo C, Riobom F, Teles L, Cruzeiro C. Necrólise Epidérmica Tóxica (Síndrome de Lyell): uma patologia para as Unidades de Queimados. Acta Med Port [Internet]. 2004 [acesso em 13 julho de 2014];17:129-40. Disponível em: file:///C:/Users/Cliente/Downloads/16572306-1-PB.pdf

14. French LE. Toxic epidermal necrolysis and Stevens Johnson syndrome: our current understanding. Allergol Int. 2006;55(1):9-16.

15. Harr T, French LE. Toxic Epidermal Necrolysis and StevensJohnson Syndrome. Orphanet J Rare Dis [Internet]. 2010 [cited 2014 July 13];5(39):1-11. Available from: http:// www.ncbi.nlm.nih.gov/pmc/articles/PMC3018455/.

16. Nagao-Dias AT, Barros-Nunes P, Coelho HLL, Sole D. Reações alérgicas a medicamentos. J Pediatr (Rio J.). 2004;80(4):259-66.

17. Sousa VD, Driessnack M, Mendes IAC. Revisão dos desenhos de pesquisa relevantes para enfermagem: Parte 1: desenhos de pesquisa quantitativa. Rev Latinoam Enferm. 2007; 15(3):502-7.

18. Fronteira I. Estudos observacionais na Era da Medicina baseada na evidência: breve revisão sobre a sua Relevância, Taxonomia e Desenhos. Acta Med Port [Internet]. 2013 [acesso em 13 julho de 2014];26(2):161-70. Disponível em: file:///C:/Users/Cliente/Downloads/3975-5347-1-PB.pdf

19. World Health Organization. Guidelines for ATC classification and DDD assignment 2014 [Internet]. Oslo (NO): WHO; 2013 [cited 2014 July 13]. Available from: http:// www.whocc.no/filearchive/publications/2014_guidelines.pdf

20. Sotelo-Cruz N, Hurtado-Valenzuela JG, Rascón-Alcantar A. Síndrome de Stevens-Johnson. Informe de 7 casos. Bol Méd Hosp Infant Méx [Internet]. 2005 [acesso em 13 de julho de 2014];62(1):51-8. Disponível em: http:// www.scielo.org.mx/scielo.php?script $=$ sci_arttext\&pid $=\mathrm{S} 1665-11462005000100005$

21. Secoli SR. Polifarmácia: interações e reações adversas no uso de medicamentos por idosos. Rev Bras Enferm. 2010;63(1):136-40.

22. Dogra A, Kaur S. Toxic Epidermal Necrolysis due to concomitant use of lamotrigine and valproic acid. Indian J Dermatol [Internet]. 2013 [cited 2014 July 13];58(5):406. Available from: http://www.ncbi.nlm.nih.gov/pmc/ articles/PMC3778793/.

23. Azulay RD, Azulay DR, Azulay-Abulafia L. Dermatologia. Rio de Janeiro (RJ): Guanabara Koogan; 2008. 